\title{
LOCAL DIOPHANTINE PROPERTIES OF SHIMURA CURVES AND THE $\overline{\mathbb{F}}_{p}$-GONALITY \\ Chuangxun Cheng
}

\begin{abstract}
In this paper, we study the local points of small degrees on Shimura curves $X_{0}^{D}(N)$ over a totally real field $F$. We then study the $\overline{\mathbb{F}}_{p}$-gonality for these Shimura curves in the case $F=\mathbb{Q}$.
\end{abstract}

Keywords: Shimura curve, gonality.

\section{Introduction}

In [5], the authors studied the local Diophantine properties of Shimura curves. In [10], the authors generalized the results in [5] and constructed Shimura curves which violates Hasse principle. In [3], the author constructed infinitely many Shimura curves which violates Hasse principle. One of the key ingredients in [3] is a careful study of local points on Shimura curves. All these papers focus on the Shimura curves over $\mathbb{Q}$. In this paper, we study the local points of small degrees on Shimura curves $X_{0}^{D}(N)$ over a totally real field $F$. We show that most of the results in section 3 of [3] still hold in totally real field case.

We also study the $\overline{\mathbb{F}}_{p}$-gonality of $X_{0}^{D}(N)$ in the case $F=\mathbb{Q}$ and give lower bound for this invariant. The reason we assume that $F=\mathbb{Q}$ is because, over a totally real field $F, X_{0}^{D}(N)$ is not geometrically integral in general and the $\overline{\mathbb{F}}_{p}$-gonality is not defined. One of the important tools we use is the inequality in Theorem 3.1, which is proved in [8].

\subsection{Curve $X_{0}^{D}(N)$}

Let $F$ be a totally real field of degree $d$. We fix an embedding $\tau_{1}: F \hookrightarrow \mathbb{R}$. Let $S_{D}$ be a finite set of finite primes of $F$ such that

$$
\left|S_{D}\right| \equiv d-1 \quad(\bmod 2) .
$$

2010 Mathematics Subject Classification: primary: 11G20 
We also denote $S_{D}$ the product of primes in this set. Let $D$ be a quaternion algebra over $F$ which is ramified at places $S_{D} \cup\left\{v \mid \infty: v \neq \tau_{1}\right\}$. D is unique up to isomorphism. We exclude the case $D=M_{2}(\mathbb{Q})$ in this paper.

Fix $\mathcal{O}_{D}$ a maximal order of $D$. If $v$ is a finite prime of $F$ such that $v \nmid S_{D}$, we fix an isomorphism $D \otimes F_{v} \cong M_{2}\left(F_{v}\right)$, under which we have $\left(\mathcal{O}_{D} \otimes \mathcal{O}_{F_{v}}\right)^{\times}=$ $G L_{2}\left(\mathcal{O}_{F_{v}}\right)$. Let $\mathcal{O}_{F}$ be the ring of integers of $F$. Let $N$ be a squarefree idea of $\mathcal{O}_{F}$ such that $\left(N, S_{D}\right)=1$. Let $\Gamma_{0}^{D}(N)$ be an open compact subgroup of $\left(D \otimes \mathbb{A}_{F}^{\infty}\right)^{\times}$ defined as follows,

$$
\Gamma_{0}^{D}(N)_{v}= \begin{cases}\left(\mathcal{O}_{D} \otimes \mathcal{O}_{F_{v}}\right)^{\times} & \text {if } v \nmid N \\
\left\{\left(\begin{array}{ll}
a & b \\
c & d
\end{array}\right) \in G L_{2}\left(\mathcal{O}_{F_{v}}\right): v \mid c\right\} & \text { if } v \mid N\end{cases}
$$

The curve $X_{0}^{D}(N)$ is the Shimura curve attached to $D$ and $\Gamma_{0}^{D}(N)$. It is a compact smooth algebraic curve over $F$ with $\mathbb{C}$-points

$$
X_{0}^{D}(N)(\mathbb{C}) \cong D^{\times} \backslash(\mathbb{C}-\mathbb{R}) \times\left(D \otimes \mathbb{A}_{F}^{\infty}\right)^{\times} / \Gamma_{0}^{D}(N)
$$

\section{2. $m$-invariant}

Let $X_{/ K}$ be a variety over a field $K$. Following [3], define the $m-$ invariant $m(X)=m\left(X_{/ K}\right)$ to be the minimum degree of a finite field extension $L / K$ such that $X(L) \neq \emptyset$.

If $K$ is a number field. Write $\Omega_{K}$ for the set of all places of $K$. For each $v \in \Omega_{K}$, we put

$$
m_{v}(X):=m\left(X_{/ K_{v}}\right)
$$

and

$$
m_{l o c}(X)=\operatorname{lcm}_{v \in \Omega_{K}} m_{v}(X) .
$$

As remarked in Remark 2.1 of [3], $m_{v}(X)=1$ for all but finitely many $v$, therefore, $m_{l o c}(X)$ is well defined.

One of the main results of this paper is the following theorem, which generalizes Theorem $8(\mathrm{~b})$ of [3].

Theorem 1.1. For our curve $X_{0}^{D}(N)_{/ F}$, we have $m_{\text {loc }}\left(X_{0}^{D}(N)\right) \mid 12$.

\subsection{Gonality}

The $K$ - gonality of a geometrically integral curve $C_{/ K}$, denoted $\gamma_{K}(C)$, is the least positive integer $n$ for which there exists a degree $n$ dominant rational map $C \rightarrow \mathbf{P}_{K}^{1}$. If $L / K$ is a field extension, we define also the $L$-gonality $\gamma_{L}(C)$ of $C$ as the gonality of $C_{L}:=C \times_{K} L$. See appendix of [8] for some general facts about gonality.

If $C_{/ K}$ has gonality $\gamma$, where $K$ is a field with finitely many elements. Then there exists a degree $\gamma$ dominant rational map $C \rightarrow \mathbf{P}_{K}^{1}$. Therefore we have the obvious inequality

$$
|C(K)| \leqslant \gamma\left|\mathbf{P}^{1}(K)\right|=\gamma(|K|+1) .
$$


So $\gamma \geqslant \frac{|C(K)|}{|K|+1}$. With this easy estimate and Theorem 3.1, we give a lower bound for $\gamma_{\overline{\mathbb{F}}_{p}}\left(X_{0}^{D}(N)\right)$ in the case $F=\mathbb{Q}$, by some counting arguments. See the corollaries in section 3 for details. In particular, we have the following result.

Theorem 1.2. Fix prime number $p$. If $N$ is squarefree, then $\gamma_{\overline{\mathbb{F}}_{p}}\left(X_{0}^{D}(N)\right) \rightarrow \infty$ as $N \rightarrow \infty$.

\subsection{Notation}

If $L$ is a perfect field we will let $\bar{L}$ denote the algebraic closure of $L$. If $L$ is a number field, we let $\mathbb{A}_{L}$ denote the ring of adeles over $L$, and $\mathbb{A}_{L}^{\infty}$ denote the ring of finite adeles over $L$. If $L=\mathbb{Q}$, we write $\mathbb{A}$ and $\mathbb{A}^{\infty}$ for $\mathbb{A}_{\mathbb{Q}}$ and $\mathbb{A}_{\mathbb{Q}}^{\infty}$, respectively.

Let $\Sigma$ be a set of primes of $F$. If a group $U$ has the form $U=\prod_{v \in \Sigma} U_{v}$, and $J$ is an ideal which is a product of some elements in $\Sigma$, we will write $U^{J}$ for the subgroup of $U$ given by $U^{J}=\prod_{v \in \Sigma, v \nmid J} U_{v}$ and $U_{J}$ for the subgroup of $U$ given by $U_{J}=\prod_{v \in \Sigma, v \mid J} U_{v}$.

In this paper, $F$ will be a totally real field. If $\mathfrak{p}$ is a finite place of $F$, we will write $F_{\mathfrak{p}}$ for the completion of $F$ at $\mathfrak{p}, \mathcal{O}_{F_{\mathfrak{p}}}$ the ring of integers of $F_{\mathfrak{p}}$, and $k_{\mathfrak{p}}$ the residue field of $\mathcal{O}_{F_{\mathfrak{p}}}$. We also write $k_{\mathfrak{p}}^{\prime}$ for the degree two extension of $k_{\mathfrak{p}}$.

\section{Local points of $X_{0}^{D}(N)$}

In this section, we apply Hensel's lemma (Proposition 2.1) to study local points on Shimura curves with $\Gamma_{0}^{D}$ level. More precisely, we compute $m_{\mathfrak{p}}\left(X_{0}^{D}(N)\right)$. The results in this section hold for general totally real field $F$. In particular, the results hold in the case $F=\mathbb{Q}$.

Proposition 2.1 (Hensel's Lemma). Let $R$ be a complete DVR with quotient field $K$ and residue field $k$. Let $X$ be a smooth algebraic variety over $K$. Suppose that $\mathcal{X}_{/ R}$ is a regular model of $X_{/ K}$. Then $X$ has a $K$-rational point if and only if the special fiber $\mathcal{X}_{/ R} \times k$ has a smooth $k$-rational point.

Proof. See for example Lemma 1.1 of [5].

\subsection{Case $\mathfrak{p} \nmid N S_{D}$}

If $\left(\mathfrak{p}, N S_{D}\right)=1$, then $X_{0}^{D}(N) / F_{\mathfrak{p}}$ has a smooth integral model $M_{0}^{D}(N) / \mathcal{O}_{F_{\mathfrak{p}}}$ which has good reduction at $\mathfrak{p}$. We consider another Shimura curve $X_{0}^{D}(\mathfrak{p} N)$ with regular integral model $M_{0}^{D}(\mathfrak{p} N) / \mathcal{O}_{F_{\mathfrak{p}}}$. The curve $M_{0}^{D}(\mathfrak{p} N) / \mathcal{O}_{F_{\mathfrak{p}}}$ has semistable reduction at $\mathfrak{p}$. The special fiber $M_{0}^{D}(\mathfrak{p} N) / k_{\mathfrak{p}}$ is isomorphic to a union of two copies of $M_{0}^{D}(N) / k_{\mathfrak{p}}$ intersecting transversely above a finite set of points $\Sigma_{0}^{D}(N)$. (See for example Theorem 10.2 of [4].) The points in $\Sigma_{0}^{D}(N)$ are called supersingular points.

Lemma 2.2. The finite set $\Sigma_{0}^{D}(N)$ is not empty. Moreover, every supersingular point is defined over $k_{\mathfrak{p}}^{\prime}$. 
Proof. Let $D^{\prime}$ be another quaternion algebra over $F$ such that $D^{\prime}$ is ramified at primes $S_{D} \cup\{\mathfrak{p}\} \cup\{v: v \mid \infty\}$. Then from section 11 of [2], we have the following bijection:

$$
\Sigma_{0}^{D}(N) \cong\left(D^{\prime}\right)^{\times} \backslash\left(D^{\prime} \otimes_{F} \mathbb{A}_{F}^{\mathfrak{p} \infty}\right)^{\times} \times F_{\mathfrak{p}}^{\times} / \Gamma_{0}^{D^{\prime}}(N)^{\mathfrak{p}} \times \mathcal{O}_{F_{\mathfrak{p}}}^{\times}
$$

Therefore, $\Sigma_{0}^{D}(N)$ is non empty (and finite).

The supersingular points are defined over $k_{\mathfrak{p}}^{\prime}$ follows from Theorem 10.2 of [4]. (See also Theorem 2.4.)

Corollary 2.3. If $\mathfrak{p} \nmid N S_{D}$, then $m_{\mathfrak{p}}\left(X_{0}^{D}(N)\right) \leqslant 2$.

Proof. This follows from the above lemma and Hensel's lemma.

\subsection{Case $\mathfrak{p} \mid N$}

The following theorem is proved in section 10 of [4]. We already used it above.

Theorem 2.4. If $\mathfrak{p} \mid N$, then the special fiber $M_{0}^{D}(N) / k_{\mathfrak{p}}$ has two irreducible components, each isomorphic to the smooth curve $M_{0}^{D}(N / \mathfrak{p}) / k_{\mathfrak{p}}$. The two irreducible components intersect transversely at the supersingular points, a corresponding point on the first copy being glued to its image under the Frobenius map.

Furthermore, at each supersingular point $x \in M_{0}^{D}(N) / k_{\mathfrak{p}}^{\prime}$, the complete local ring is isomorphic to $W\left(k_{\mathfrak{p}}^{\prime}\right)[1 / p][[X, Y]] /\left(X Y-\varpi_{\mathfrak{p}}^{a_{x}}\right)$ for some positive integer $a_{x}$.

Corollary 2.5. If $\mathfrak{p} \mid N$, then $m_{\mathfrak{p}}\left(X_{0}^{D}(N)\right) \leqslant 4$.

Proof. The proof is the same as the proof of Proposition 14 of [3]. We sketch it here. Let $z \in M_{0}^{D}(N)_{k_{\mathfrak{p}}^{\prime}}$ be a point coming from a supersingular point in $M_{0}^{D}(N / \mathfrak{p})\left(k_{\mathfrak{p}}^{\prime}\right)$. Then the complete local ring at $z$ is isomorphic to $W\left(k_{\mathfrak{p}}^{\prime}\right)[1 / p][[X, Y]] /\left(X Y-\varpi_{\mathfrak{p}}^{a_{z}}\right)$ for some positive integer $a_{z}$. If $a_{z}>1$, then we have to blow up $z\left(a_{z}-1\right)$ times to obtain a regular model. This procedure produces $\left(a_{z}-1\right)$ rational curves over $k_{\mathfrak{p}}^{\prime}$. Each such rational curve has $\left(\left|k_{\mathfrak{p}}^{\prime}\right|-1\right)$ smooth points over $k_{\mathfrak{p}}^{\prime}$, which lift to give rational points of $X_{0}^{D}(N)$ over $K_{0}=\operatorname{Frac}\left(W\left(k_{\mathfrak{p}}^{\prime}\right)\right)$.

If $a_{z}=1$, let $K=K_{0}(\sqrt{\varpi})$ be a ramified quadratic extension of $K_{0}$. Let $R$ be the ring of integers of $K$. Then the complete local ring of $z \in M_{0}^{D}(N)_{/ R}$ is isomorphic to $R[[X, Y]] /\left(X Y-(\sqrt{\varpi})^{2}\right)$. The same argument as above shows that $X_{0}^{D}(N)$ has rational points over $K$. Since $\left[K: F_{\mathfrak{p}}\right]=4$, the statement follows.

\subsection{Case $\mathfrak{p} \mid S_{D}$}

We recall the $p$-adic uniformization theory for Shimura curves. Let $v$ be a finite place of $F$ at which $D$ is ramified. Let $P$ be an open compact subgroup of $(D \otimes$ $\left.\mathbb{A}^{\infty}\right)^{\times}$such that $P_{v}=\left(\mathcal{O}_{D} \otimes_{F} \mathcal{O}_{F_{v}}\right)^{\times}$. We have a Shimura curve $X_{P}$ (with level $P)$ which is defined over $F$. 
Let $F_{v}^{u r}$ be the maximal unramified extension of $F_{v}$, let $\Omega_{F_{v}}$ be Drinfeld's upper half plane. Consider the analytic space $\Omega_{F_{v}}^{n r}=\Omega_{F_{v}} \otimes_{F_{v}} F_{v}{ }^{n r}$ over $F_{v}$. We let $g \in G L_{2}\left(F_{v}\right)$ act on $\Omega_{F_{v}}^{n r}$ via the natural (left) action on $\Omega_{F_{v}}$ and the action of $F_{r o b} F_{v}$ val (det $\left.g\right)$ on $F_{v}{ }^{u r}$. We also let $n \in \mathbb{Z}$ act on $\Omega_{F_{v}}^{n r}$ through the action of $F r o b_{F_{v}}^{-n}$ on $F_{v}{ }^{n}$. This gives an $F_{v}$-rational action of $G L_{2}\left(F_{v}\right) \times \mathbb{Z}$ on $\Omega_{F_{v}}^{n r}$. Moreover, the $F_{v}$-analytic space $G L_{2}\left(F_{v}\right) \backslash\left(\Omega \times\left(P^{v} \backslash\left(D \otimes \mathbb{A}_{F}^{\infty v}\right)^{\times} / D^{\times}\right)\right)$algebraizes canonically to a scheme $\mathfrak{X}_{P}$ over $F_{v}$. Let $X$ and $\mathfrak{X}$ be the inverse limits of $X_{P}$ and $\mathfrak{X}_{P}$ over all $P$. Then a special case of Theorem 5.3 of [11] gives the following theorem. (See also section 1 of [6].)

Theorem 2.6. There exists $a\left(D \otimes \mathbb{A}_{F}^{\infty v}\right)^{\times} \times \mathbb{Z}$-equivariant, $F_{v}$-rational isomorphism

$$
X \otimes_{F} F_{v} \cong \mathfrak{X} .
$$

In particular, we have an $F_{v}$-rational isomorphism

$$
X_{P} \otimes_{F} F_{v} \cong \mathfrak{X}_{P}
$$

Furthermore, there exists an integral model $M_{P}$ for $X_{P}$ over $\mathcal{O}_{F_{v}}$, and the above isomorphism can be extended to schemes over $\mathcal{O}_{F_{v}}$.

Corollary 2.7. If $\mathfrak{p} \mid S_{D}$, then $m_{\mathfrak{p}}\left(X_{0}^{D}(N) \leqslant 2\right.$.

Proof. In our case, we have $M_{P}=X_{0}^{D}(N) / \mathcal{O}_{F_{\mathfrak{p}}}, \mathfrak{X}_{P}=\mathfrak{X}_{0}^{D}(N)$. The special fiber of $\mathfrak{X}_{0}^{D}(N) / \mathcal{O}_{F_{\mathfrak{p}}}$ has non-degenerate quadratic singular points. The dual graph $\mathfrak{G}$ attached to the special fiber of $\mathfrak{X}_{0}^{D}(N) / \mathcal{O}_{F}$ is the quotient

$$
G L_{2}\left(F_{\mathfrak{p}}\right)^{+} \backslash\left(\Delta \times\left(\Gamma_{0}^{D}(N)^{\mathfrak{p}} \backslash\left(D \otimes \mathbb{A}_{F}^{\infty \mathfrak{p}}\right)^{\times} / D^{\times}\right)\right),
$$

where $\Delta$ is the well-known tree attached to $S L_{2}\left(F_{\mathfrak{p}}\right)$. The singular points of $\mathfrak{X}_{0}^{D}(N) \times k_{\mathfrak{p}}$ correspond to the edges of $\mathfrak{G}$.

To prove the corollary, it suffices to find a smooth $k_{\mathfrak{p}}^{\prime}$-rational point on $M_{0}^{D}(N) \cong_{k_{\mathfrak{p}}^{\prime}} \mathfrak{X}_{0}^{D}(N)$. Because every vertex of $\mathfrak{G}$ has degree at most $\left|k_{\mathfrak{p}}\right|+1$, every irreducible component $\mathfrak{X}_{i}$ (which is isomorphic to $\mathbf{P}^{1}$ ) of $\mathfrak{X}_{0}^{D}(N) / k_{\mathfrak{p}}$ has at most $\left|k_{\mathfrak{p}}\right|+1$ singular points, hence at least $\left|k_{\mathfrak{p}}\right|^{2}+1-\left(\left|k_{\mathfrak{p}}\right|+1\right)$ smooth $k_{\mathfrak{p}}^{\prime}-$ rational points.

From the above analysis, we have the following result.

Corollary 2.8. For any finite prime $\mathfrak{p}$ of $F, X_{0}^{D}(N)\left(k_{\mathfrak{p}}^{\prime}\right)$ is non empty.

\section{3. $\overline{\mathbb{F}}_{p}$-gonality of $X_{0}^{D}(N)$}

If $F \neq \mathbb{Q}, X_{0}^{D}(N)$ is not geometrically integral in general. From now on, we assume that $F=\mathbb{Q}$.

In Theorem 1.1 of [1], the author gives a linear lower bound on the $\mathbb{C}$-gonality of Shimura curves. In [3], the author uses this bound and some other ingredients 
to show that almost all Shimura curves $X_{0}^{D}(N)$ are potentially Hasse principle violation. In this section, we study the $\overline{\mathbb{F}}_{p}$-gonality of Shimura curve $X_{0}^{D}(N)$. More precisely, we give lower bounds of the $\overline{\mathbb{F}}_{p}$-gonality of Shimura curve $X_{0}^{D}(N)$ by an idea of [8] and some counting arguments. The following theorem is proved in Theorem 2.5 of [8].

Theorem 3.1. Let $X$ be a geometrically integral curve over a perfect field $K$. Let $L \supset K$ be an algebraic field extension. Assume that $X(K) \neq \emptyset$. Then $\gamma_{L}(X) \geqslant$ $\sqrt{\gamma_{K}(X)}$.

By Corollary 2.8, $X_{0}^{D}(N)\left(\mathbb{F}_{p^{2}}\right)$ is non empty for any $p$. We may apply this theorem to give a lower bound for $\gamma_{\overline{\mathbb{F}}_{p}}\left(X_{0}^{D}(N)\right)$ by computing $\gamma_{\mathbb{F}_{p^{2}}}\left(X_{0}^{D}(N)\right)$.

\subsection{Case $p \nmid N S_{D}$}

We count the number of $\mathbb{F}_{p^{r}}$-rational points of $X_{0}^{D}(N) / \mathbb{F}_{p}$. To do this, we follow the approach of [5]. Let $p$ be a prime number not dividing $N S_{D}$. The EichlerShimura relation leads to the following formula for the Zeta-function of $X_{0}^{D}(N)_{/ \mathbb{F}_{p}}$.

$$
Z\left(X_{0}^{D}(N)_{/ \mathbb{F}_{p}}, t\right)=\frac{\operatorname{det}\left(1-T_{p} t+\langle p\rangle p t^{2}\right)}{(1-t)(1-p t)}
$$

Here $T_{p}$ is the Hecke operator acting on the space $H^{0}\left(X_{0}^{D}(N), \Omega^{1}\right),\langle p\rangle$ is the diamond operator. Set $T_{1}=I d, T_{p^{-1}}=0$, then we have identity

$$
T_{p^{r+1}}=T_{p^{r}} T_{p}-\langle p\rangle p T_{p^{r-1}} .
$$

By taking $\frac{d}{d t} \log$ on both sides of the Zeta-function, we have the following equality for $r \geqslant 1$ :

$$
\left|X_{0}^{D}(N)\left(\mathbb{F}_{p^{r}}\right)\right|=1+p^{r}+\operatorname{Trace}\left(\langle p\rangle p T_{p^{r-2}}-T_{p^{r}}\right) .
$$

In our setting, for $\left(n, N S_{D}\right)=1$, Trace $\left(T_{n}\right)$ can be computed in a very explicit way. We state the following theorem which is a special case of Theorem 6.8.4 of [7].

Theorem 3.2 (Eichler-Selberg trace formula). Let $\chi$ be a Dirichlet character $(\bmod N), S_{2}\left(\Gamma_{0}^{D}(N), \chi\right)$ be the space of forms of weight two, level $\Gamma_{0}^{D}(N)$, and character $\chi$. Then we have the following formula.

$$
\begin{aligned}
\operatorname{Trace}\left(T_{n}\right) & :=\operatorname{Trace}\left(T_{n} \mid S_{2}\left(\Gamma_{0}^{D}(N), \chi\right)\right) \\
& =\frac{1}{12} \chi(\sqrt{n}) N \prod_{p \mid N}\left(1+p^{-1}\right) \prod_{p \mid S_{D}}(p-1)-\sum_{t} a(t) \sum_{f} b(t, f) c(t, f) .
\end{aligned}
$$

Here each term is as follows.

(1) $\chi(\sqrt{n})=0$ if $n$ is not a square.

(2) $t$ runs over all integers such that $t^{2}-4 n$ are negative or square. For such $t, a(t)$ is some number depends on $n$ and $S_{D}$. 
(3) $f$ runs over all positive divisors of $m$, where $m$ is a positive integer given by $t^{2}-4 n=m^{2} d$ if $d \neq 0$; otherwise $m=1$. For such an $f, b(t, f)$ is some number depends on $n$ and $S_{D}, c(t, f)$ is some number depends on $n, S_{D}$, and $N$.

For the explicit formulaes of $a(t), b(t, f)$, and $c(t, f)$, see Theorem 6.8.4 of [7]. To compute equation (3.1), we have $\chi=1$.

Corollary 3.3. If $p \nmid N_{S} D$, then

$$
\gamma_{\mathbb{F}_{p^{2}}}\left(X_{0}^{D}(N)\right) \geqslant \frac{1+p^{2}+\operatorname{Trace}\left(\langle p\rangle p-T_{p^{2}}\right)}{p^{2}+1} .
$$

Here, the right hand side of the inequality can be computed by Eichler-Selberg trace formula, denote it by $G_{2}\left(p, N, S_{D}\right)$. We then have

$$
\gamma_{\overline{\mathrm{F}}_{p}}\left(X_{0}^{D}(N)\right) \geqslant \sqrt{G_{2}\left(p, N, S_{D}\right)} .
$$

Proof. The first inequality follows from the fact that

$$
\left|X_{0}^{D}(N)\left(\mathbb{F}_{p^{2}}\right)\right| \leqslant \gamma_{\mathbb{F}_{p}^{2}}\left(X_{0}^{D}(N)\right)\left|\mathbf{P}^{1}\left(\mathbb{F}_{p^{2}}\right)\right|=\gamma_{\mathbb{F}_{p}^{2}}\left(X_{0}^{D}(N)\right)\left(p^{2}+1\right) .
$$

The second inequality follows from Theorem 3.1.

Remark 3.4. In the above computation, we did not write down all the terms explicitly. The reason is that the formulaes in Theorem 6.8.4 of [7] are complicated, even in the simple case where $N$ is a prime number. In this case, from equation (5) of [10], we have

$$
\left|X_{0}^{D}(N)\left(\mathbb{F}_{p^{2}}\right)\right|=\Sigma_{2}(N)-p \Sigma_{0}(N)+\frac{1}{12}(N+1)(p-1) \prod_{d \mid S_{D}, d \text { prime }}(d-1) .
$$

Here $\Sigma_{m}(M)$ are some number defined at the beginning of section 1.3 of [10], which are related to $a, b$, and $c$ in Eichler-Selberg trace formula. In particular, $\Sigma_{2}(N)-p \Sigma_{0}(N) \geqslant 0$ by Proposition 1.3 of [10]. Therefore we have the following lower bound of $\gamma_{\mathbb{F}_{p^{2}}}\left(X_{0}^{D}(N)\right)$.

$$
\gamma_{\mathbb{F}_{p^{2}}}\left(X_{0}^{D}(N)\right) \geqslant \frac{1}{12\left(p^{2}+1\right)}(N+1)(p-1) \prod_{d \mid S_{D}, d \text { prime }}(d-1) .
$$

Note that we have $12\left(p^{2}+1\right)$ as the denominator. See Proposition 3.1 of [8] for a similar result in the case of modular curves.

Corollary 3.5. Fix a prime $p$. If $N$ is square free and $p \nmid N S_{D}$, then

$$
\gamma_{\mathbb{F}_{p^{2}}}\left(X_{0}^{D}(N)\right) \geqslant \frac{1}{12\left(p^{2}+1\right)}(p-1)\left(n_{N}+1\right) \prod_{d \mid S_{D}, d \text { prime }}(d-1)
$$


where $n_{N}$ is the maximal prime divisor of $N$. Therefore,

$$
\gamma_{\overline{\mathbb{F}}_{p}}\left(X_{0}^{D}(N)\right) \geqslant \sqrt{\frac{1}{12\left(p^{2}+1\right)}(p-1)\left(n_{N}+1\right) \prod_{d \mid S_{D}, d \text { prime }}(d-1) .}
$$

Proof. There exists a dominant rational map $X_{0}^{D}(N) \rightarrow X_{0}^{D}(N / n)$ for any prime divisor $n$ of $N$. By Proposition A.1 of [8], $\gamma_{\mathbb{F}_{p^{2}}}\left(X_{0}^{D}(N)\right) \geqslant \gamma_{\mathbb{F}_{p^{2}}}\left(X_{0}^{D}(N / n)\right)$. The corollary follows from the computation in Remark 3.4.

\subsection{Case $p \mid N$}

We have the following inequality

$$
\gamma_{\mathbb{F}_{p^{2}}}\left(X_{0}^{D}(N)\right) \geqslant \frac{\left|X_{0}^{D}(N)\left(\mathbb{F}_{p^{2}}\right)\right|}{p^{2}+1}
$$

Note that $X_{0}^{D}(N)_{/ \mathbb{F}_{p}}$ is a union of two copies of a smooth curve $X_{0}^{D}(N / p)_{/ \mathbb{F}_{p}}$ intersect at supersingular points, which are rational over $\mathbb{F}_{p^{2}}$. The set of supersingular points is bijective to the following double quotient

$$
\bar{D}^{\times} \backslash\left(\bar{D} \otimes \mathbb{A}^{\infty p}\right)^{\times} \times \mathbb{Q}_{p}^{\times} / \Gamma_{0}^{\bar{D}}(N / p)^{p} \mathbb{Z}_{p}^{\times}
$$

Here $\bar{D}$ is another definite quaternion algebra over $\mathbb{Q}$ obtained by changing the local invariants of $D$ at $p$ and $\infty$. This double quotient is a finite set. Let $\sigma\left(p, N / p, S_{D}\right)$ be the number of elements of this set. We have the following result.

Corollary 3.6. If $p \mid N$, then

$$
\gamma_{\mathbb{F}_{p^{2}}}\left(X_{0}^{D}(N)\right) \geqslant 2 G_{2}\left(p, N / p, S_{D}\right)-\frac{\sigma\left(p, N / p, S_{D}\right)}{p^{2}+1} .
$$

Therefore,

$$
\gamma_{\overline{\mathbb{F}}_{p}}\left(X_{0}^{D}(N)\right) \geqslant \sqrt{2 G_{2}\left(p, N / p, S_{D}\right)-\frac{\sigma\left(p, N / p, S_{D}\right)}{p^{2}+1}} .
$$

Notice that $G_{2}\left(p, N / p, S_{D}\right) \geqslant \frac{\sigma\left(p, N / p, S_{D}\right)}{p^{2}+1}$. Therefore

$$
2 G_{2}\left(p, N / p, S_{D}\right)-\frac{\sigma\left(p, N / p, S_{D}\right)}{p^{2}+1} \geqslant G_{2}\left(p, N / p, S_{D}\right) \geqslant \frac{\sigma\left(p, N / p, S_{D}\right)}{p^{2}+1}>0 .
$$

Remark 3.7. Since $N$ is square free, we have an explicit bound for $G_{2}\left(p, N / p, S_{D}\right)$. The same argument as in the case $p \nmid S_{D} N$ gives us the following inequality.

$$
\gamma_{\mathbb{F}_{p^{2}}}\left(X_{0}^{D}(N)\right) \geqslant \frac{1}{12\left(p^{2}+1\right)}(p-1)\left(n_{N / p}+1\right) \prod_{d \mid S_{D}, d \text { prime }}(d-1) .
$$




\subsection{Case $p \mid S_{D}$}

Let $\bar{D}$ be another definite quaternion algebra over $\mathbb{Q}$ obtained by changing the local invariants of $D$ at $p$ and $\infty$. By Proposition 4.4 of [9], the set of irreducible components of $\mathfrak{X}_{0}^{D}(N)$ is bijective to the set of vertices of its dual graph, which is bijective to two copies of the following double quotient

$$
\bar{D}^{\times} \backslash\left(\bar{D} \otimes \mathbb{A}^{\infty}\right)^{\times} / \Gamma_{0}^{\bar{D}}(N) .
$$

This is a finite set. Let $v\left(p, N, S_{D}\right)$ be the number of elements of this double quotient. The set of singular points of $\mathfrak{X}_{0}^{D}(N)_{/ \mathbb{F}_{p}}$ is bijective to the set of edges of its dual graph, which is bijective to the following double quotient

$$
\bar{D}^{\times} \backslash\left(\bar{D} \otimes \mathbb{A}^{\infty}\right)^{\times} / \Gamma_{0}^{\bar{D}}(N p) .
$$

This is also a finite set. Let $e\left(p, N, S_{D}\right)$ be the number of elements of this set.

From the proof of Corollary 2.7, the number of smooth $\mathbb{F}_{p^{2}}$ points on $\mathfrak{X}_{0}^{D}(N)$ is at least $2\left(p^{2}-p\right) v\left(p, N, S_{D}\right)$. The number of rational $\mathbb{F}_{p^{2}}$ points on $\mathfrak{X}_{0}^{D}(N)$ is at least $2\left(p^{2}-p\right) v\left(p, N, S_{D}\right)+e\left(p, N, S_{D}\right)$. We have the following result.

Corollary 3.8. If $p \mid S_{D}$, then

$$
\gamma_{\mathbb{F}_{p}^{2}}\left(X_{0}^{D}(N)\right) \geqslant \frac{2\left(p^{2}-p\right) v\left(p, N, S_{D}\right)+e\left(p, N, S_{D}\right)}{p^{2}+1} .
$$

Therefore,

$$
\gamma_{\overline{\mathbb{F}}_{p}}\left(X_{0}^{D}(N)\right) \geqslant \sqrt{\frac{2\left(p^{2}-p\right) v\left(p, N, S_{D}\right)+e\left(p, N, S_{D}\right)}{p^{2}+1}} .
$$

\section{References}

[1] D. Abramovich,A Linear Lower Bound on the Gonality of Modular Curves, Internat. Math. Res. Notices 1996, no. 20, 1005-1011.

[2] M. Carayol, Sur la mauvaise réduction des courbes de Shimura, Compositio Math. 59 (1986), no. 2, 151-230.

[3] P. Clark, On the Hasse principle for Shimura curves, Israel J. Math. 171 (2009), no. 1, 349-365.

[4] F. Jarvis, Mazur's Principle for totally real fields of odd degree, Compositio Mathematica 116 (1999) 39-79.

[5] B.W. Jordan, B. Livne, Local diophantine properties of Shimura curves, Math. Ann. 270 (1993), 385-390.

[6] B. Jordan, B. Livne, Y. Varshavsky, Local points on p-adically uniformized Shimura varieties.

[7] T. Miyake, Modular Forms. Springer-Verlag, 2006

[8] B. Poonen, Gonality of modular curves in characteristic p, Math. Res. Lett. 14 (2007), no. 4, 691-701. 
[9] K.A. Ribet, On modular representations of $\operatorname{Gal}(\bar{Q} / Q)$ arising from modular forms. Invent. Math. 100 (1990), no. 2, 431-476.

[10] A. Skorobogatov, A. Yafaev, Descent on certain Shimura curves. Israel J. Math. 140 (2004), 319-332.

[11] Y. Varshavsky, p-adic uniformization of unitary Shimura varieties. J. Differential Geometry 49 (1998), 75-113

Address: Chuangxun Cheng: Department of Mathematics, Bielefeld University, Germany.

E-mail: ccheng@math.uni-bielefeld.de

Received: 18 October 2012; revised: 26 October 2012 